\title{
El regadío de Torrent y su evolución a lo largo del siglo XX
}

\author{
José Luís Fresquet Gozalvo \\ Departamento de Producción Vegetal. Universitat Politècnica de València \\ jfresque@prv.upv.es
}

Resumen. Para poder entender el estado actual del regadío en el término municipal de Torrent, hay que remontarse a los primeros años del siglo XX en los que permanecía con las mismas características de su diseño inicial alrededor del siglo XI y estudiar las sucesivas ampliaciones y modificaciones que fue experimentando a lo largo del siglo. Los cambios que se produjeron y sobre todo el aumento de la superficie regada no fue uniforme, los primeros años son de gran lentitud y encaminados principalmente al aprovisionamiento de agua para compensar el caudal que ha sido sustraído por el Ayuntamiento para consumo de la población. A mitad de siglo toman mayor velocidad con nuevos alumbramientos y ampliación de la huerta y, será en el último tercio cuando se va a producir el mayor crecimiento de la superficie regada.

El factor limitante del regadío de Torrent ha sido tradicionalmente la falta de agua que en nuestro caso se vio agravada a lo largo de los siglos por el gran crecimiento demográfico que competía en la utilización del recurso. La huerta antigua de Torrent a principios de siglo se puede cifrar en unas 4.000 hanegadas, de las que 3.600 se encontraban junto al núcleo urbano y la restantes en la zona oeste del término y bastante separadas de la población en las partidas de la Masía del Juez y de La Horteta. Las mejoras que se fueron incorporando al regadío torrentito fueron de dos tipos, el primero comprendía las medidas destinadas a aumentar las disponibilidades de agua y a disminuir las pérdidas en la red de riego y el segundo que comprendía la incorporación de las nuevas técnicas de riego. La Comunidad de Regantes de Torrent, constituida en 1903 fue la primera entidad impulsora de estas medidas y tuvo una gran actividad hasta mitad del siglo. En la segunda mitad fueron las numerosas Sociedades de regantes la que se mostraron más activas. El resultado fue muy positivo y el regadío de Torrent pasó de 4.000 a más de 40.000 hanegadas a finales de siglo.

Palabras clave: Azud, acequia, pozo de riego, grupo motobomba, regadío.

\begin{abstract}
In order to understand the current situation of the irrigation in the municipality of Torrent, the first years of the 20th century must be revisited, as the same characteristics of the initial design of around the 11th century remained. Besides, the following extensions and modifications suffered throughout the century must be studied. The changes that happened and especially the increase of irrigated surface were not uniform. The first years of development were really slow and mostly aimed at the water supplies in order to compensate the flow withdrawn by the city council with consumption purposes. Towards the middle of the century the process speeded up with new lightening and the extension of vegetable gardens and in the third part of the century, the biggest increase of irrigated surface took place.

The limiting condition was worsened throughout the centuries due to the population growth that competed for the use of the resource. The old greenbelt of Torrent in the early century is estimated to have 350 hectares, part of them nearby the metropolitan area and the rest in the western side of the municipality, rather far from the population in the lands of Masía del Juez and La Horteta. The improvements that were added to the irrigation in Torrente were of two different kinds, the first one consisted of the measures aimed at increasing the water availability and the reduction of losses in the irrigation system and the second one comprised the inclusion of new irrigating technologies. The community of Regantes de Torrent, established in 1903, was the first entity to drive these measures and had great activity up to the middle of the century. In the second half, the numerous partnerships of regantes were more active. The result was very positive and the irrigation of Torrent turned from 350 hectares to 3,500 in the late century.
\end{abstract}

Keywords: Dam, channel, well used for irrigation, pumping unit, irrigated land. 


\section{Introducción}

El presente trabajo describe el regadío del término municipal de Torrent, tanto el antiguo como el desarrollado a lo largo del pasado siglo, en el que se han incorporado nuevos alumbramientos de agua, nuevos sistemas de embalse y conducción, y nuevos sistemas de riego a presión. También se examina la variación que ha tenido lugar tanto en la superficie regada como en el tamaño de las parcelas.

El término municipal de Torrent se encuentra situado en el extremo sur-oeste de la huerta de Valencia. Tiene una superficie de $69,56 \mathrm{~km}^{2}$, que equivalen a 6.956 ha y a 83.472 hanegadas. Tiene forma trapezoidal con una longitud media de $16 \mathrm{~km}$ y una anchura media de $6 \mathrm{~km}$. Se extiende de este a oeste desde una llanura que supone una extensión natural de la huerta de Valencia, hasta una zona abrupta que constituye la Sierra Perenxisa, cuyas laderas de levante y mediodía pertenecen al mismo. Las lomas del Vedat, Morredondo, Barret i Faxardet modelan una superficie irregular y componen una orografía que se completa con los barrancos de Torrent, de Poyo, Horteta, la Bota, els Gils, las Cañas así como las cañadas de Alonso y Godelleta.

\section{El regadío de la huerta antigua o histórico}

La primera referencia histórica del riego de la huerta torrentina se encuentra en la Carta Puebla otorgada en 1248 por el Maestre de la Orden de San Juan del Hospital, señor de la villa tras la conquista del Reino de Valencia, en la que se reparte a los nuevos colonos traídos de Cataluña, una superficie de "tres jovadas de huerta y una casa con un huerto de una hanegada" y se impone la condición de mantener el azud y la acequia bien limpios", con lo que se prueba que el regadío ya estaba establecido en época musulmana y que, tanto el azud como las acequias de reparto, estaban construidas y en funcionamiento desde hace más de setecientos cincuenta años.

El origen del agua que ha permitido la subsistencia y el desarrollo de Torrent, hay que buscarlo en el barranco o torrente que seguramente dio el nombre a la villa y concretamente en el barranco llamado de la Horteta, de las Fuentes o de Cortixelles que, de todas maneras se llama según el tramo que se considere. Nace en el término de Turis y atraviesa el de Torrent de Oeste a Este en toda su longitud, unos $16 \mathrm{kms}$. Este barranco posee numerosas fuentes en sus márgenes que permiten una continuidad en su caudal a lo largo del año, muy inferior al que se presenta en las grandes lluvias en las que ejerce el papel de desagüe de las mismas hacia el lago de la Albufera.

Las fuentes que permanecen a principios del siglo XX, enumerándolas de arriba abajo son: la Font de la Teula, la de Manyes, la de la Bota, la del Sapo, la de Baylón, la de la Carrasquera y la de Sant Luis. Esta última es la más importante en caudal y no solo contribuye al regadío, sino que ha sido canalizada en todo su trayecto, más bien entubada, y proporciona el agua de boca de la población, que no olvidemos que en esos momentos ascendía a 8.561 habitantes.

Además de las fuentes, existen unas filtraciones que emergen en el mismo lecho del barranco y dan lugar a pozas o clots como el de Bailón o el de Rosafina de tamaño considerable y que apenas varían en nivel.

La zona de la Horteta está dividida en dos subzonas por el camino de Xarcos-secs: l'Horteta de Dalt y l'Horteta de Baix. El agua que riega a ambas, se deriva del cauce del barranco mediante un pequeño azud que se encuentra a unos 300 metros aguas arriba de la fuente de Bailón. La acequia de tierra viene por el lateral norte del cauce, ganando cota hasta l'Horteta de Dalt, 
transcurre por la parte alta de la misma en sentido Sur-Norte, y de ella salen tres brazales en sentido Este que conducen el agua a las pequeñas acequias de riego. La superficie regada es de 50 hanegadas. La acequia principal, para pasar el camino, tiene un pequeño acueducto de 3 metros de alto y 12 de largo que conduce el agua a la cota superior de l'Horteta de Baix. El primer campo que puede regar es de algarrobos centenarios que nunca han sido regados, a continuación empieza la zona regada de unas 60 hanegadas que se extiende entre el camino, el barranco y el pie de sierra.

Todas las acequias son de tierra y las obras más importantes de este riego son el azud y el acueducto. El primero es un pequeño muro de 1 metro de alzada y 3 de longitud realizado con piedras tomadas con mortero de cal que, partiendo del centro del cauce, se dirige a la orilla Norte derivando a la acequia un pequeño caudal. El acueducto, aunque pequeño, tiene su gracia por lo bien empotrado que se encuentra en ambos extremos en el talud de tierra natural, por sus arcos rebajados tipo carpanel y por la pequeña acequia que lo corona y que era suficiente para el escaso caudal que conducía. Está realizado en fábrica de piedras tomadas con mortero de cal y el tamaño de las mismas es mayor en la base que en las de coronación.

Este pequeño sistema hidráulico estuvo funcionando hasta que se instalaron los pozos de El miracle de la Horteta..., y en el año 1973, como consecuencia de la pavimentación del camino de Xarcos-secs y debido a la reducida altura del acueducto, que limitaba el paso de los vehículos, fue derribado en la parte que limitaba con el mismo, quedando en buen estado el arco situado al Norte.

La huerta del Mas de Jutge es una zona de forma triangular, de unas 200 hanegadas de superficie, que limita por su base con el barranco de la Horteta, por el lado Este con el barranco del Zahorí y por el lado Oeste con el camino de Xarcos-secs, el sistema hidráulico está formado por el azud de la Horteta, la Sequieta del Camp, el acueducto de los Arquillos que supera el barranc de l'Horteta, sendas balsas de regulación y una red de acequias que distribuyen el agua en las 200 hanegadas perfectamente niveladas para facilitar la mejor distribución del agua de riego.

El azud de derivación se encuentra aproximadamente en el mismo lugar en que se inician los riegos de l'Horteta de Dalt. Tiene una longitud de unos 8 metros y una altura de 1'50 en la parte central del barranco. Está hecho de mampostería de piedra tomada con mortero de cal y tenías unos tableros de madera para facilitar el paso del agua a la acequia.

La Sequieta del Camp iniciaba su recorrido en el azud y, siguiendo el margen Norte del barranco, iba ganando cota hasta llegar a al camino de Xarcos-secs en donde, mediante un pequeño sifón, atravesaba el camino y pasaba al margen Sur que seguía hasta llegar a la Font de la Carrasquera, donde volvía a atravesar el camino, esta vez con una cubierta de losas y seguía el cauce hasta superar el meandro que hace el barranco en esta zona para dirigirse, siguiendo las curvas de nivel, hasta el encuentro del camino de la Contienda con el de Xarcossecs. De allí se dirigía a la Caseta del Pollastre y de ésta hacia el acueducto para superar el barranco. A continuación, volvía a atravesar el camino y por su margen derecho se dirigía hacia la Masía; metros antes de la llegada, volvía a atravesar el camino y vertía sus aguas en una gran balsa de regulación. A continuación, volvía a atravesar el camino y llegaba a una pequeña balsa situada en el lado Oeste del cementerio; de esta balsa volvía a salir e iniciaba una red de acequias que permitían el riego de las 200 hanegadas. En su tramo final constituía un pequeño desagüe que permitía verter los sobrantes en el Barranc de Zahorí.

La sección de la acequia era de 50 x $60 \mathrm{~cm}$. excepto en la zona intermedia en la que, para mantener el nivel, alcanzaba profundidades superiores al metro e incluso al metro cincuenta centímetros. 
El recorrido de la acequia es de unos 6 kilómetros de longitud y es de tierra en todo su recorrido, lo que obligaba a proceder a su limpieza o monda todos los años, labor que era realizada por los agricultores que se beneficiaban de las misma y que debían aportar un número de jornales proporcional al número de hanegadas que regaba cada uno para mantener el cauce en condiciones. La limpieza se llevaba a afecto en la época de menor trabajo, que generalmente coincidía con el invierno y, tanto por su longitud como por su profundidad, suponía una penosa labor.

El acueducto de los Arquillos es la parte más sobresaliente de esta conducción hidráulica. El cauce del barranco, en este punto, es amplio y profundo, por lo que el acueducto es de dimensiones notables. Su longitud es de 50 metros y su altura en el eje del barranco supera los 5 metros. Consta de seis arcos de distinto tamaño, más anchos los dos centrales y más estrechos los cuatro laterales. Este acueducto, como consecuencia de las grandes avenidas de agua sufridas, se ha venido abajo en la parte central en varias ocasiones. Este sistema hidráulico dejó de funcionar en los años setenta.

El regadío más importante es el que afecta a las inmediaciones de la población y se extiende por el Norte y Este del núcleo urbano, comprendiendo las partidas de Xenillet, Benisaet, Acequia de Picanya, Corberà, Safranar y Răfol. El sistema hidráulico consta de Azud, Acequia General, con dos acueductos para superar sendas vaguadas, Acequias Secundarias y Red de Riego.

El Azud de derivación es el elemento más espectacular y se encuentra situado en el Barranco, a 4 kilómetros aguas arriba del antiguo casco urbano, en el paraje conocido como el Pantano. Tiene una longitud de 67 metros y una altura máxima de 5 metros, presentando en su margen izquierdo una caseta para la toma de derivación con una compuerta metálica de apertura manual. La presa tiene en su parte central un amplio vierteaguas de 31 metros de largo y 9 metros de ancho, con una pendiente para facilitar el desagüe.

Se encuentra totalmente colmatado por los muchos años con que cuenta y por las aguas tan cargadas de lodos que circulan en las frecuentes avenidas, por lo que hace muchos años que no se produce el menor almacenamiento de aguas. A este propósito, conviene recordar el episodio ocurrido a mediados del siglo XIX en el que el Ayuntamiento, acuciado por las demandas de los agricultores que pedían se aumentase el caudal disponible para el riego, contrató un Proyecto de Recrecimiento de la Presa y procedió a la ejecución del mismo con gran dispendio para las arcas municipales. Se encargaron de ambos, unos arquitectos de Valencia que garantizaron las obras por un espacio de un año. La casualidad hizo que durante ese periodo no se produjera ninguna avenida, pero a los pocos meses se produjo una, de no mucha intensidad, y toda la obra construida se vino abajo, quedando incólume la vieja fábrica. La decepción del Ayuntamiento y de los labradores fue enorme ya que vieron frustrarse todas sus esperanzas.

$\mathrm{El} A q u d$ sólo cuenta con un muro de caja en la parte Sur que protege el margen, la caseta y el canal de derivación, pero no tiene muro de contención en la parte Norte, ni losa inferior, por lo que se ha producido una gran erosión en ambas zonas, erosión que incluso pone en peligro la estabilidad del mismo y que sería necesario corregir para asegurar su longevidad.

La Acequia General es un canal de tierra, a principio de siglo, que, partiendo del azud y siguiendo el margen Sur del barranco, conduce el agua hasta el núcleo urbano, en su camino tiene que superar dos obstáculos, el primero a escasos metros de su nacimiento, es la desembocadura del barranco de las Cañas, donde tiene un pequeño acueducto de $25 \mathrm{~m}$ de longitud, formado por dos arcos de $10 \mathrm{~m}$ cada uno, el segundo a un kilómetro del primero para superar la vaguada de San Gregorio, de $33 \mathrm{~m}$ de longitud, que sólo tiene un arco, por lo que se llama El Arquello. En el que penetra por el final de la calle de la Ermita y 
discurre por la misma hasta llegar a la Plaza del Canónigo, donde tuerce a la izquierda para coger la calle de la Acequia, (hoy Gómez Ferrer), hasta llegar a la calle de la Purificación, donde desvía a la derecha en dirección al Molino de Represa que existe al Este de la población $\mathrm{y}$ al que suministra un caudal suficiente para el accionamiento del mismo.

Esta Acequia, dentro del casco urbano es de obra y está cubierta por losas en una buena parte de su recorrido, pero el tramo inicial es de tierra y no se hace de obra hasta que se terminan las minas en 1914. La Acequia General, desde su entrada al pueblo, va derivando unos ramales secundarios, las acequias de Xenillet, Corberá, Benisaet, Jucarancha o Safranar, Ráfol, Picanya y Llengües (Desagüe al barranco). Cada una de estas acequias, tenía una zona propia de riego que coincide con el nombre de las antiguas partidas del Término Municipal.

Podemos pensar que el principal objetivo de esta conducción era el suministro urbano, en un tiempo en el que no había otra posibilidad de suministro y en el que la población fue aumentando desde unos pocos cientos a varios miles de habitantes. Otro objetivo importante era el accionamiento del molino de trigo para el servicio de la población, siendo necesario que llegase al mismo el máximo caudal y, en tercer lugar, su utilización para el regadío de las mejores tierras del Término Municipal que, precisamente, se encuentra en esta zona.

Tras la última derivación, la Acequia General, siguiendo el margen del camino, se dirige hacia el Barrranco, convirtiéndose en un desagüe para cuando un exceso de lluvias no interesara para el riego y fuera conveniente derivarlo de nuevo al Barranco. En este tramo tóma el nombre de Acequia de les Llengües.

Este sistema hidráulico, según las Ordenanzas de la Comunidad de Regantes de Torrent, en 1903, en su artículo 3', "La Comunidad puede disponer, para su aprovechamiento, de todas las aguas que van a parar al Pantano, que viene a ser, aproximadamente, mil seiscientos veinte litros por minuto".

Así mismo, en su artículo 4' expresa que "Tienen derecho al uso de las aguas que posee la Comunidad para su aprovechamiento en riego, las tres zonas denominadas Benisaet, Acequia de Picaña y Zafranar, que están contiguas y ocupan una extensión de tres mil trescientas noventa y seis hanegadas y media".

A simple vista observamos la gran desproporción entre el caudal disponible de $1620 \mathrm{l} / \mathrm{m}$ y las tres mil cuatrocientas hanegadas que tienen derecho a riego, lo cual nos hace suponer que una parte importante de esa superficie no se regaba y que sus propietarios las dedicaban a otros cultivos, como indica el nombre de alguna subpartida llamada Els Moscatells, en alusión al cultivo de esta variedad de viñedo.

Así pues, la Huerta de Torrent antigua a principios del siglo XX tenía un gran déficit de agua en gran parte provocado por la canalización de la Fuente de San Luis para traer agua potable a Torrent, que tuvo lugar en 1892. 


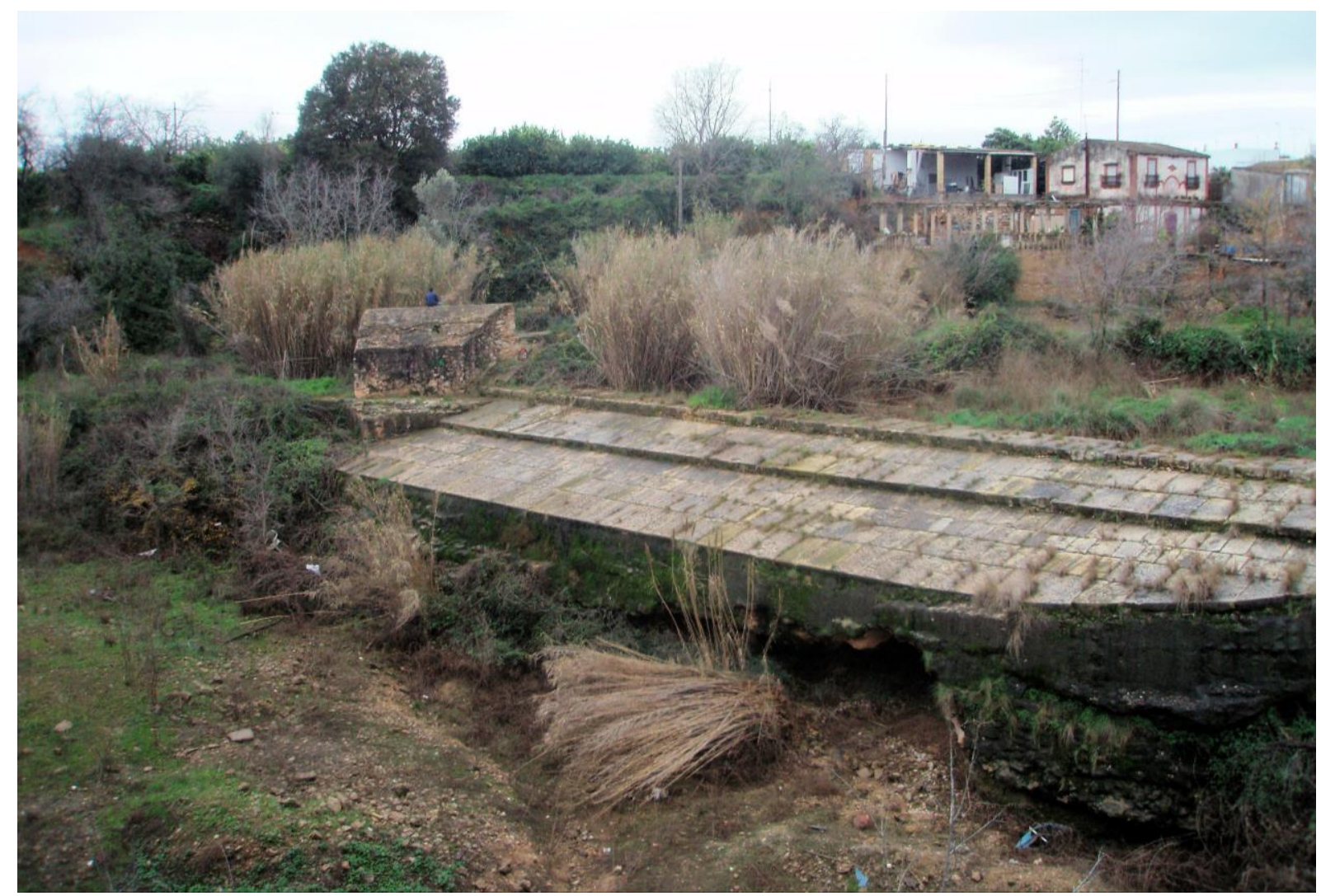

Fig.1. Azud del barranco de l'Horteta.

\section{Consolidación del regadío antiguo}

Ante este panorama, la nueva Comunidad de Regantes toma la decisión de aumentar el caudal de agua disponible y encarga a su Sindicato que inicie los estudios correspondientes para conseguir este fin. El Sindicato contrata los servicios del ingeniero D. José $M^{a}$ Bordás, que realiza el Primer Proyecto de Acrecentamiento de Aguas del regadío torrentino partiendo de una galería o mina que se excavaría en el extremo Noroeste del Término Municipal, partida de La paret decantá, donde se había realizado un pozo de prueba, Pou de Manifest, en el que, después de cortar varias capas de margas arcillosas y algunos cantos rodados, se llegó a la profundidad de14 metros, donde se encontró terreno de aluvión formando el manto freático, que pudo comprobarse que era de importancia. Se practicaron después las operaciones de nivelación que dieron por resultado que el agua podía, sin necesidad de aparatos de elevación, llegar naturalmente al Pantano.

Aprobado el Proyecto, en 1904, comenzaron los trabajos de perforación de la galería siguiendo la capa acuífera encontrada y construyéndose, así mismo, treinta pozos lumbreras y de desescombro. La galería se prolongó hasta una longitud de $530 \mathrm{~m}$, consiguiéndose un caudal de $2.700 \mathrm{l} / \mathrm{m}$.

Los excelentes resultados obtenidos con la primera mina, animaron a la Comunidad que en 1.912 encargó al ingeniero D. Manuel Rodrigo un nuevo estudio de captación de aguas, que centró en la Partida de la Contienda, más próxima al Pantano que el anterior, y utilizando los mismos procedimientos, se llegó al nivel freático a los $12 \mathrm{~m}$ de profundidad y se realizó una galería de $500 \mathrm{~m}$, con los correspondientes pozos lumbreras, obteniéndose un caudal de $2.8801 / \mathrm{m}$. Esta galería desembocaba junto al Azud. 
En este segundo alumbramiento, se practicaron cuatro sondeos en busca de aguas artesianas y a una profundidad de 20 metros, se encontró una segunda capa freática artesiana que produjo un caudal supletorio de $1.1881 / \mathrm{m}$, siendo el caudal total de $4.0681 / \mathrm{m}$.

Como las galerías excavadas corrían el peligro de derrumbarse, hubo que asegurarlas en las paredes laterales y el techo con sendos muretes y una bóveda que al principio se hicieron con atobones y luego se optó por el hormigón armado.

El coste de las obras de ambos alumbramientos fue de unas 40.000 pesetas y se obtuvo un aumento de caudal de $6.7681 / \mathrm{m}$. Este importe fue totalmente sufragado por los regantes de la Comunidad, proporcionalmente a la superficie que tenía cada uno.

Terminadas las minas, la Comunidad de Regantes decidió convertir la Acequia General, que era de tierra, en un canal de obra cubierto que evitara las filtraciones que se producían en lecho y cajeros, así como la evaporación natural en tan largo recorrido. El nuevo cauce tenía una longitud de $3.800 \mathrm{~m}$ y con su ejecución se dio un nuevo paso para asegurar el suministro de agua a la Huerta Antigua.

Para ampliar las miras del regadío torrentino a principios de 1920, hemos recurrido a los datos que se aportaron al III Congreso Nacional de Riegos, celebrado en Valencia en 1921, una de cuyas Ponencias se dedicaba exclusivamente a los "Pequeños Regadios", que los definían como "Aquellos que dotados de un caudal de aguas reducido, se establece por particulares a titulo civil o basados en la concesión de una mina o de aguas subálveas".

Para este Congreso, la Federación Valenciana de Sindicatos Agrícolas preparó un Ensayo de Estadística de los alumbramientos de agua para los pequeños riegos de la Provincia de Valencia, y en él aparece nuestro municipio con doce pozos y dos minas. De los doce pozos, hay seis accionados con máquina de vapor, cuatro con gas pobre y dos eléctricos. Las profundidades en la mayoría son de $18 \mathrm{~m}$, y sólo los accionados por motor eléctrico llegan a 35 y 49 metros respectivamente. El caudal de la mayoría es de $1.500 \mathrm{l} / \mathrm{m}$ y el caudal total que suministran es de $15.100 \mathrm{l} / \mathrm{m}$, lo que supone el doble del caudal de que disponía la Comunidad de Regantes después de realizar los nuevos alumbramientos de agua.

Los pozos a que hace referencia la Federación Valenciana de Sindicatos, son con toda probabilidad los siguientes:

- $\quad$ Pozo del Mas de Don Pedro. En la masía del mismo nombre.

- $\quad$ Pozo de Santonja. En la finca del mismo nombre sita en el camino viejo de Alacuás.

- $\quad$ Pozo de Pavía. En la masía del mismo nombre.

- $\quad$ Pozo de la finca de nuestra Señora del Remedio, en el Alter.

- $\quad$ Alumbramiento Buenos Aires, en el Mas de Caboteta.

- $\quad$ Pozo de la Muntanyeta de Cabrera, en el Vedat.

- $\quad$ Pozo de Sant Jordi, en el Safranar.

- $\quad$ Pozo dels Sants de la Pedra o Pou del Ceg, en la partida Acequia de Picaña.

- $\quad$ Pozo de San Roque o Vaporet dePedroso, en la partida del Alter. 


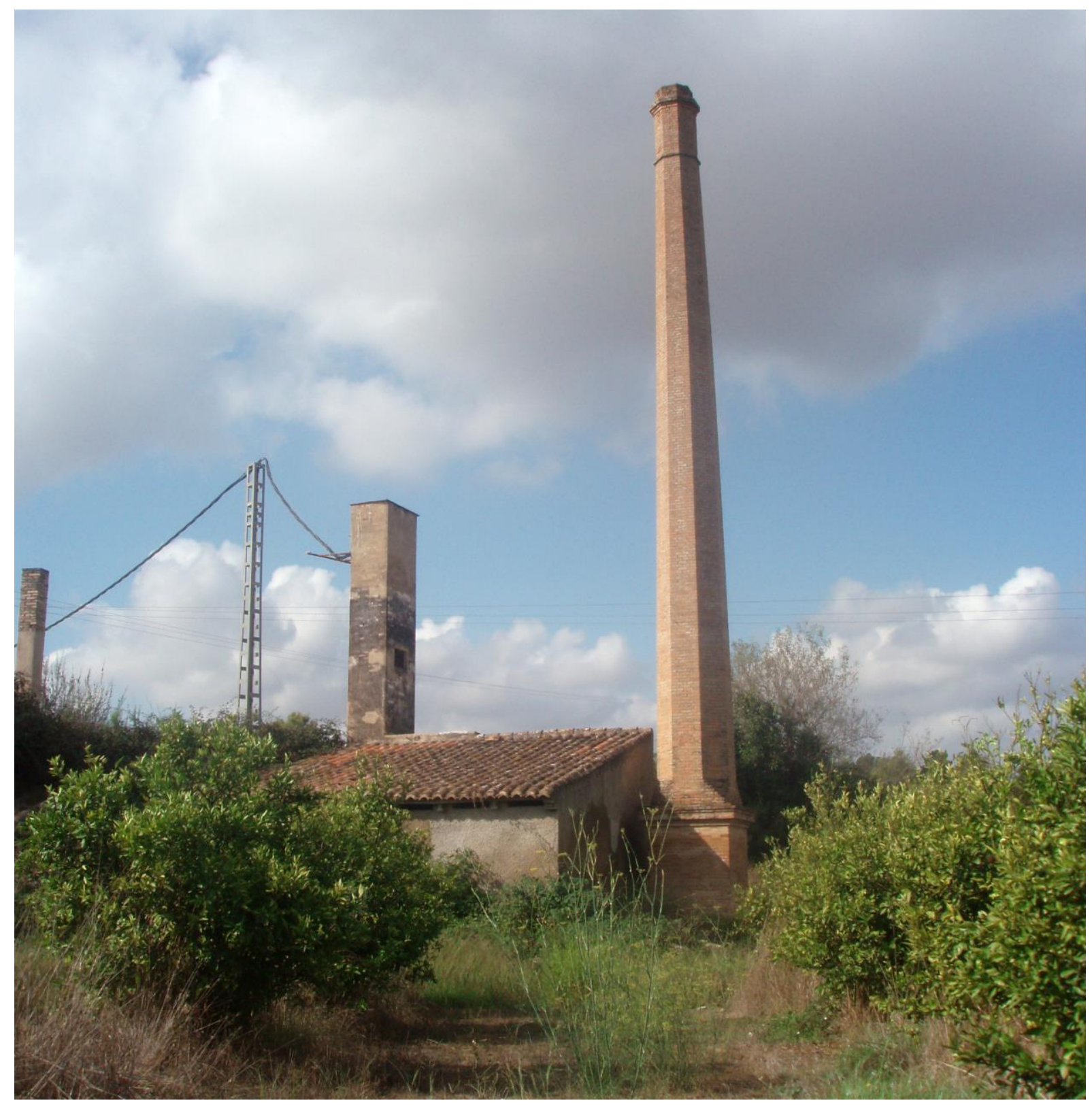

Fig. 2. Pozo de riego en la Masía de Pavía

La mayoría son particulares, pertenecientes a ricos propietarios que los utilizan en sus fincas, aunque algunos venden horas de agua a los vecinos. Los tres últimos pozos pertenecen a Sociedades de Regantes que empiezan a constituirse en estas fechas y que en los próximos años, cuarenta y cincuenta, serán las que lideren la apertura de pozos que transformarán nuestro término municipal. Observamos también como estos tres pozos van a regar la Huerta Antigua, signo evidente de las necesidades de agua de la misma, a pesar del incremento de caudal que han supuesto las nuevas minas excavadas.

De las acequias secundarias derivaban los brazales que iban repartiendo el agua a las acequias de riego propiamente dichas. Como la huerta estaba establecida siguiendo las curvas de nivel, y los puntos más altos se encontraban cerca del núcleo urbano y los más bajos en el linde con el término de Picaña, los brazales tenían dirección Oeste-Este, mientras que las acequias de riego la tenían Norte-Sur, de manera que el agua de riego iba 
siguiendo la pequeña pendiente de los bancales a medida que los inundaba. Los dos brazales más importantes salen de la Acequia del Ráfol y son el de la Barraca y el de Perales.

En principio, toda la red hidráulica era de canales o acequias de tierra, poco a poco se fueron transformando en canales de ladrillo y hormigón, primero los importantes y más tarde el resto. Las acequias de Benisaet y Jucarancha como circulaban, en parte, por el núcleo urbano fueron las primeras en hacerse de obra. La acequia de Picaña se hizo de ladrillo y hormigón en el periodo de la Guerra Civil, la del Ráfol en los años cuarenta, etc.

Todavía se harán nuevas Sociedades de Regantes en esta huerta, antes de iniciar la transformación del secano en regadío, y surgen los pozos de San Cristóbal (1924) primero en el lado Sur del Barranco y luego en el Norte, Santa Rita (1927) y El Omet (1930) en el Safranar. La Comunidad de Regantes, en 1937, excava un pozo en La Contienda, junto a la segunda mina, para aumentar el caudal disponible, es el pozo del Sindicat Vell, también denominado Virgen del Pilar 1.

\section{Nuevos regadíos}

Hemos visto como hasta finales de los años treinta, todos los esfuerzos de la Comunidad de regantes a través de su Sindicato, y de varios grupos de agricultores formando Sociedades de Regantes, van encaminados al suministro de agua para la Huerta Antigua. Es verdad que propietarios particulares han hecho alumbramientos en sus fincas o masías y las han puesto en regadío, pero las superficies regadas no son importantes.

En los años cuarenta y cincuenta es cuando se instalan los nuevos regadíos, merced al gran impulso que llevan a cabo nuestros agricultores constituyendo hasta veintisiete nuevas Sociedades de Regantes que extraerán y llevarán sus aguas a distintos puntos del secano torrentino.

En 1940, en la partida de La Foya, a espaldas del cementerio, se abre el pozo de Santa Fe, que va a permitir el riego en esta zona de secano. Del mismo año es el pozo de La Pelleria, en el Tollo, que en poco tiempo se queda seco.

En 1941, se abren dos nuevos pozos en El Alter, cerca del de Pedroso, zona que ya ha demostrado tener una capa freática potente, son el de San Jaime y el de San Isidro, como la zona más inmediata es la huerta antigua que no necesita más agua, se conduce el agua en tubería de hormigón enterrada, hasta la Foya. No obstante, a su paso por el Safranar se colocan válvulas o compuertas que permiten el riego en la zona en caso de necesidad. En el mismo año se excava con éxito el pozo del Ráfol o de San Rafael, propiedad del dueño de la finca, el Marqués de la Romana, que da un caudal de 3.000 litros por minuto y que contribuye al riego de la misma.

En 1942, en La Contienda se excavan dos pozos, uno particular el Pozo de Sancho, un rico propietario que lo utilizará para su finca, el otro, el Pozo de San Luís Beltrán de una Sociedad que rápidamente pondrá en regadío un millar de hanegadas. Por otro lado en la partida de Don Jeroni se abre el pozo de la Mare de Deu dels Desamparats también de una sociedad de regantes que por el escaso caudal sólo ponen en regadío un centenar de hanegadas. En la partida Mas Don Pedro, en el T.M de Aldaya se constituye la Sociedad de San Valero, cuyo pozo da un caudal de 4.500 litros por minuto y pone en regadío cerca de 2.000 hanegadas, la mayor parte de Torrent. En la partida del Alter, próximo a San Isidro, pero en T.M de Picaña, se excava el Pozo de San Ramón que con un caudal de 3.500 litros por minuto se conducirá en tubería enterrada a la partida de La Foya. 
En 1944, la Cooperativa del Campo, en la partida de La Punxa, excava el Pozo de San Salvador, también conocido como el Pou de la Pedra. Tiene un caudal de 3.000 litros por minuto y pone en regadío un millar de hanegadas.

En 1945, el Sindicato de Riegos de Torrent lleva a cabo un nuevo pozo en la partida de La Contienda, cerca del anterior y de la vieja mina, se obtiene un caudal de 3.000 litros por minuto que se incorpora a la Acequia General y se distribuye por la Huerta Antigua. En la partida del Tollo, una sociedad de regantes excava un pozo que con un caudal de 3.500 litros por minuto permite la puesta en regadío de 1.200 hanegadas. En la partida de La Punxa, en el T.M de Aldaya, se abre el pozo de la Mare de Deu dels Dolors que con un caudal de 3.000 litros por minuto permite la puesta en regadío de 1.700 hanegadas.

En 1946, se excavan dos pozos, uno particular en el Mas de Covatelles que obtuvo un caudal de $1.000 \mathrm{l} / \mathrm{m}$ que permitió la puesta en riego de una pequeña parte de la masía, y otro en la partida del Alter, el Pozo Nuevo del Alter, de una sociedad de regantes, que con un caudal de $2.000 \mathrm{l} / \mathrm{m}$ permitió la puesta en riego de 500 hanegadas.

Vemos pues que en menos de una década la nueva superficie que se puede poner en riego es de unas 13.000 hanegadas, cuatro veces superior a la Huerta Antigua. Lo cual no quiere decir que en este período se llevasen a cabo todas las transformaciones de secano en regadío que eran factibles por la disponibilidad de agua.

En los años cincuenta, sigue la excavación de pozos al mismo ritmo que en la década anterior, y así tenemos:

- En 1950, apertura del pozo de San Antonio de Padua en la partida del Alter, junto al camino, con un caudal de $1.800 \mathrm{l} / \mathrm{m}$ que permite el riego de unas 500 hanegadas.

- Del mismo año es el pozo de San Nicolás de Bari situado en la partida de la Alberca, junto al camino Real, se obtiene un caudal de $2000 \mathrm{l} / \mathrm{m}$ y pone en regadío unas 700 hanegadas. Este pozo siempre ha tenido problemas ocasionados por la arena del agua.

- En 1951, la agrupación de regantes La Providencia, cuyos miembros son propietarios en la partida Mas de Don Pedro, excavan un pozo en el término de Chiva, partida del Maset Roig, lindante con nuestro término y obtienen un caudal de $3.000 \mathrm{l} / \mathrm{m}$ que les permite poner en regadío unas mil hanegadas. El presidente de la agrupación es el Sr. Tomás Baviera, dueño de la masía del mismo nombre, que se asegura así la dotación de agua que no le puede suministrar el viejo pozo de su masía.

- En 1953, la Sociedad Civil de Regantes Viernes Santo, conocedora de la riqueza de aguas freáticas del Pla, excava un pozo en la partida de La Punxa, en término de Aldaya, lindante con el nuestro, y obtiene un caudal de $2.500 \mathrm{l} / \mathrm{m}$ que le permite regar una superficie de 1.000 hanegadas. En perforaciones posteriores obtuvo un caudal de más de $4.000 \mathrm{l} / \mathrm{m}$ que le permitió llevar el agua mediante una tuberia enterrada de más de nueve kilómetros hasta la Foya donde riega unas 2.000 hanegadas.

- En 1954, la Agrupación de Regantes Pozo San Francisco, en la partida Mas de Don Pedro, excavó un pozo que dio un caudal de $3.600 \mathrm{l} / \mathrm{m}$, que les permitió poner en regadío unas 2.000 hanegadas en la misma zona.

- En 1955, la Sociedad de Regantes Motor de Morredondo, en la partida del mismo nombre y situado a los pies de la colina, excavó un pozo que dio un caudal de 2.000 $1 / \mathrm{m}$, que les permitió poner en regadío un millar de hanegadas. 
- En 1956, la Comunidad de Regantes San Paulino, en la partida de la Contienda, cerca del barranco, excavó un pozo obteniendo un caudal de $3.000 \mathrm{l} / \mathrm{m}$ que les permitió poner en riego unas 1.500 hanegadas.

- En 1961, la Sociedad Civil Santos Patronos, después de sendos fracasos en la búsqueda de agua, excavó un pozo en la partida Mas de Don Pedro, junto al camino de los Arquillos y obtuvo un pequeño caudal que no llegaron a explotar. Posteriormente lo volvieron a intentar con éxito en la partida de La Punxa, término de Aldaya y obtuvieron un caudal de $3.000 \mathrm{l} / \mathrm{m}$, como su zona de interés era la primera, construyeron una tubería que unía ambos pozos.

- En 1964, la Sociedad Civil de Regantes San Vicente Ferrer puso en funcionamiento un pozo, que se había excavado en 1944, en la partida de La Alberca, obteniendo un caudal de $3.000 \mathrm{l} / \mathrm{m}$, que puso en riego un millar de hanegadas.

- En 1968, se excavó otro pozo con el mismo nombre, en la partida de la Alberca, relativamente cerca del anterior, promovido por Morredondo Torrent Ltda. S.A.T. $\mathrm{n}^{\circ}$ 7205. Que obtuvo un caudal de $3.0001 / \mathrm{m}$ y que permitió poner en riego unas 3.000 hanegadas.

- En 1979, la Sociedad Civil de Regantes El Miracle de l’Horteta, realizó una perforación en la partida Cañada de Alonso y obtuvo un caudal de $2.600 \mathrm{l} / \mathrm{m}$ que permitió poner en regadío unas mil hanegadas.

Esta relación incompleta expresa claramente el interés demostrado por los agricultores en los alumbramientos de aguas que se ha mantenido constante a lo largo del siglo. También hubo muchos fracasos, unos totales cuando no se encontraba agua y, otros parciales cuando el pozo que en principio tenía agua, con el tiempo de uso o con los periodos de sequía, descendía de nivel o se agotaba, pero el empeño de los agricultores y las nuevas tecnologías aplicadas, en forma de máquinas perforadoras de gran rendimiento y de gran capacidad para profundizar, y la nueva generación de bombas sumergibles o bombas buzo, hizo que a la postre se obtuviera un gran éxito en el alumbramiento de aguas.

En 1980, la Confederación Hidrográfica del Júcar, terminó las obras del magnífico canal Júcar - Turia, que partiendo del pantano de Tous conduce sus aguas a la nueva planta potabilizadora de Picassent y a la vieja de Manises. Aunque el proyecto inicial, parece ser, sólo tenía esta finalidad, la necesidad de agua para riego de todas las comarcas por las que pasaba y el empeño y constancia de todos los agricultores a través de las sociedades de regantes, de las Cámaras Agrarias Locales y Provincial, de los sindicatos de agricultores, etc, consiguieron que todos los excedentes de agua para consumo humano se dedicaran al riego. En Torrent se consiguieron dos tomas, la doce, compartida con otros pueblos de l'Horta Sud y la trece, compartida con Alacuas y Aldaya. Se constituyeron las sociedades de regantes de cada toma, con la particularidad de que los socios no son particulares, sino algunas de las sociedades de regantes ya existentes, con lo que se facilita extraordinariamente el funcionamiento de la entidad.

En principio, el canal no ha venido a poner en regadío nuevas tierras, ya que el agua va destinada a las tierras situadas en su margen derecha, que ya habían sido transformadas en regadío, pero ha servido para afianzar el regadío de las mismas que en épocas de gran consumo y en períodos de sequía, habían visto peligrar la continuidad de sus cultivos.

En los años ochenta, el secano torrentino se había reducido al extremo occidental del término municipal, la Cañada de Alonso, la de Godelleta, la de Falcó, Barbeta y Casablanca, los intentos que se habían realizado hasta el momento de alumbramiento de aguas habían fracasado, pero en 1989 y 1990, dos nuevos pozos van a cambiar la situación. 
El de la Sierra, promovido por la Comunidad de Regantes Pozo de la Sierra, en la partida de Esgarravaques, que obtuvo un caudal de más de $8.000 \mathrm{l} / \mathrm{m}$, que le ha permitido poner en riego unas 6.000 hanegadas.

El de los Giles, promovido por la Sociedad Civil de Regantes Pozo Els Xils, en la partida Cañada del Corral, que obtuvo un caudal de $6.400 \mathrm{l} / \mathrm{m}$ y que le ha permitido poner en regadío unas 8.000 hanegadas.

El último pozo perforado con éxito ha sido el de la Inmaculada, promovido por la Sociedad Civil Riego Agrícola Alumbra, en la partida de la Contienda, en 1995, que con un caudal de $2.500 \mathrm{l} / \mathrm{m}$ permite regar un millar de hanegadas, que no eran de secano pero que tenían dificultades de suministro de agua.

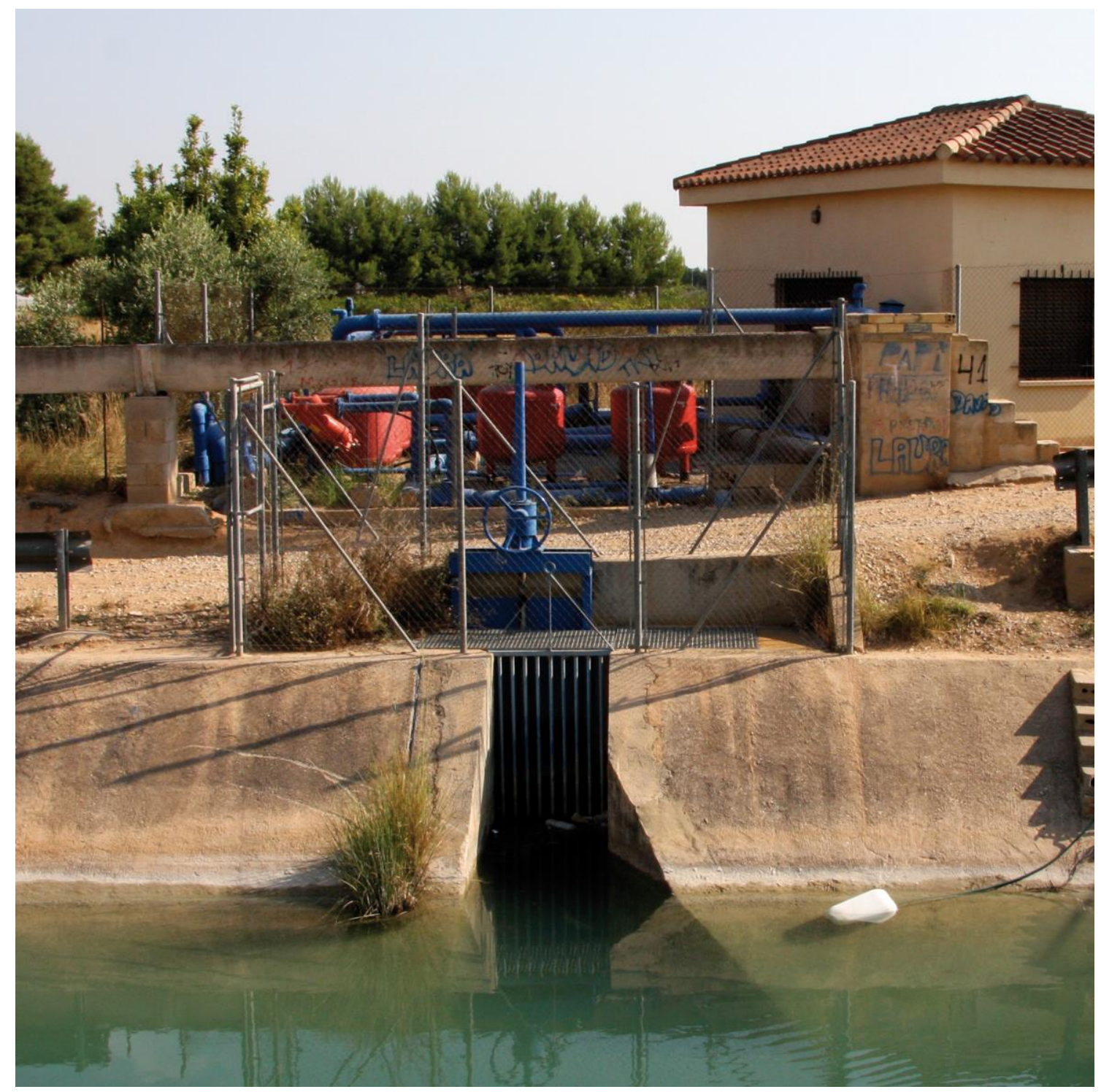

Fig. 3. Cabezal de riego localizado junto al canal del Júcar 
Tabla 1. Relación de balsas y depósitos existentes.

\begin{tabular}{|l|r|r|}
\hline BALSA & POLÍGONO & PARCELA \\
\hline Depósito de los Giles & 30 & 9072 \\
\hline Depósito Miracle de la Horteta & 30 & 747 \\
\hline Balsa del Morenito & 50 & 116 \\
\hline Balsa de Sancho & 52 & 89 \\
\hline Depósito de San Luis & 50 & 213 \\
\hline Depósito de San Paulino & 50 & 8 \\
\hline Depósito de Santos Patronos & 2 & 286 \\
\hline Depósito de Sierra uno & 2 & 47 \\
\hline Depósito de Sierra dos & 2 & 47 \\
\hline Balsa de Morredondo & 22 & 93 \\
\hline Balsa de San Vicent Ferrer & 22 & 138 \\
\hline Balsa Marchadella & 20 & 6 \\
\hline Balsa de Nuestra Señora de los Desamparados & 14 & 44 \\
\hline Balsa de los Santos de la Piedra & Picaña & Picaña \\
\hline Balsa de Covatelles & 47 & 40 \\
\hline
\end{tabular}

Tabla 2. Relación de Pozos.

\begin{tabular}{|c|c|c|c|c|c|c|c|c|}
\hline NOMBRE & POLIGONO & PARCELA & $A N ̃ O$ & \begin{tabular}{|l} 
SUPERFICIE \\
REGADA \\
\end{tabular} & \begin{tabular}{|l|} 
AFORO \\
INICIAL \\
\end{tabular} & PROFUNDID AD & UTMX & UTMY \\
\hline Posada del Pavo & 47 & 40 & 1880 & & 50 & 10 & 713514 & 4365145 \\
\hline Noria de Torán & 0 & 0 & 1899 & 0 & 0 & 0 & 719378 & 4368179 \\
\hline Del Sordo & 9 & 62 & 1902 & 8 & 800 & 40 & 717186 & 4369062 \\
\hline Mas de Don Pedro & 4 & 8 & 1905 & 0 & 1000 & 36 & 713102 & 4368746 \\
\hline Sant Jordi & 13 & 256 & 1912 & 67 & 65 & 190 & 720394 & 4367912 \\
\hline Santonja & 9 & 167 & 1912 & & & & 718369 & 4369633 \\
\hline Santos de la Piedra & 11 & 14 & 1912 & & & & 720210 & 4368703 \\
\hline $\begin{array}{l}\text { El Pedroso o Sant } \\
\text { Roc } \\
\end{array}$ & 13 & 174 & 1915 & 25 & 2500 & 150 & 718778 & 4369668 \\
\hline San Cristóbal & & & 1924 & & & & 719512 & 4369062 \\
\hline Pou de Santonja & 62 & 30 & 1930 & & 500 & 60 & 713750 & 4366532 \\
\hline L'Omet & 13 & 412 & 1930 & 33 & 500 & 48 & 719534 & 4367310 \\
\hline Sindicat Vell & 52 & 107 & 1937 & & 3000 & 45 & 714368 & 4367778 \\
\hline Santa Fe & 41 & 177 & 1940 & & 2500 & 45 & 718544 & 4367396 \\
\hline Motor de La Pelleria & 62 & 70 & 1940 & 8 & 2000 & 80 & 714878 & 4367150 \\
\hline Sant Jaume & 10 & 8 & 1941 & 58 & 2500 & 150 & 718978 & 4369875 \\
\hline Pou de Colau & 35 & 10 & 1941 & & 1000 & 35 & 715624 & 4368549 \\
\hline El Ràfol & 14 & 55 & 1941 & 50 & 3000 & 40 & 719668 & 4366869 \\
\hline San Luís Bertrán & 51 & 81 & 1942 & 108 & 3000 & 40 & 713588 & 4367532 \\
\hline $\begin{array}{l}\text { Mare de Deu dels } \\
\text { Desemparats }\end{array}$ & 7 & 69 & 1942 & 8 & 1800 & 65 & 715412 & 4368801 \\
\hline Santa Rita de Cassia & 12 & 348 & 1942 & 100 & 2800 & 125 & 719938 & 4368111 \\
\hline El Tollo & 61 & 137 & 1945 & 100 & 3500 & 125 & 714108 & 4366488 \\
\hline Pou de les Porcateres & 38 & 119 & 1945 & & 100 & 40 & 718701 & 4367633 \\
\hline Sindicat Nou & 51 & 124 & 1945 & & 3000 & 45 & 714051 & 4367774 \\
\hline Pou Nou de l'Alter & 9 & 181 & 1946 & 40 & 2000 & 180 & 717626 & 4369582 \\
\hline Mas de Covatelles & 46 & 22 & 1946 & 25 & 1000 & 47 & 713529 & 4364431 \\
\hline Sant Antoni de Padua & 8 & 31 & 1950 & 3 & 1800 & 110 & 717073 & 4368932 \\
\hline Sant Nicolau de Bari & 22 & 254 & 1950 & 58 & 2000 & 90 & 714641 & 4366234 \\
\hline
\end{tabular}




\begin{tabular}{|c|c|c|c|c|c|c|c|c|}
\hline La Providencia & 4 & 42 & 1951 & 100 & 3000 & 180 & 711803 & 4368581 \\
\hline Fernández & 6 & 24 & 1952 & 7 & 3000 & 80 & 714019 & 4368865 \\
\hline San Francisco & 32 & 36 & 1954 & 155 & 3600 & 160 & 712018 & 4367902 \\
\hline Muntanyeta Cabrera & 54 & 229 & 1954 & & & & 716232 & 4367420 \\
\hline San Isidro & 10 & 12 & 1954 & & & & 718883 & 4369482 \\
\hline San Ramón & & & 1954 & & & & 719256 & 4369228 \\
\hline San José & & & 1954 & & & & 719813 & 4369052 \\
\hline Sancho & 52 & 20 & 1954 & & & & 714342 & 4367539 \\
\hline Morredondo & 22 & 76 & 1955 & 100 & 4000 & 198 & 714657 & 4365562 \\
\hline Marsilla & 30 & 229 & 1956 & 8 & & 300 & 711936 & 4366600 \\
\hline Desamparados & 14 & 49 & 1956 & 25 & 4000 & 100 & 719500 & 4366963 \\
\hline San Paulino & 51 & 150 & 1956 & 125 & 3500 & 150 & 713035 & 4367547 \\
\hline Viernes santo & 0 & 0 & 1956 & 0 & 0 & 0 & 713415 & 4368876 \\
\hline Santos Patronos & 32 & 118 & 1961 & 160 & & 310 & 712433 & 4367499 \\
\hline Miracle de l'Horteta & 30 & 49 & 1961 & 58 & 2500 & 150 & 710025 & 4365790 \\
\hline Vives & 56 & & 1962 & & & & 717675 & 4367618 \\
\hline La Alberca & 45 & 140 & 1964 & 42 & 3000 & 160 & 715147 & 4366113 \\
\hline Motor de Rosafina & 30 & 66 & 1965 & 17 & 3000 & 120 & 708990 & 4365627 \\
\hline Calicanto & 2 & 129 & 1966 & & & 180 & 709996 & 4366320 \\
\hline Pou de la Fontsanta & 22 & 302 & 1967 & 8 & 800 & 90 & 714521 & 4364184 \\
\hline San Vicente Ferrer & 22 & 255 & 1968 & 3500 & 3000 & 125 & 714927 & 4365698 \\
\hline San Enrique & 22 & 20 & 1975 & 83 & 3500 & 200 & 714430 & 4365878 \\
\hline Motor de Paltrot & 1 & 83 & 1984 & 83 & 450 & 173 & 707256 & 4366806 \\
\hline San Pascual & 45 & 11 & 1986 & & 2500 & 150 & 715566 & 4365802 \\
\hline Motor de Corell & 2 & 250 & 1986 & 8 & 3000 & 140 & 711471 & 4367565 \\
\hline Canyada de l'Albarder & 20 & 23 & 1988 & & 3000 & 60 & 717211 & 4364296 \\
\hline El Morenito & 50 & 116 & 1988 & 17 & 2500 & 80 & 713712 & 4367241 \\
\hline La Serra & 2 & 53 & 1989 & 501 & 8496 & 314 & 707428 & 4366858 \\
\hline Els Gils & 30 & 509 & 1990 & 665 & 6421 & 300 & 709461 & 4364925 \\
\hline Inmaculada & 52 & 71 & 1995 & 81 & 2500 & 203 & 713557 & 4367796 \\
\hline
\end{tabular}

\section{Sociedades de regantes}

Como terminamos de ver, los agricultores de Torrent a lo largo del siglo han venido formando grupos, comunidades, asociaciones, en definitiva constituyendo Sociedades Civiles de Regantes con el objeto de proceder al alumbramiento de aguas que les permitieran poner en regadío sus fincas de secano, o de asegurarse el suministro constante de agua a sus fincas de regadío.

La constitución de estas sociedades se iniciaba por unos pocos miembros, que tenían sus fincas contiguas o próximas y que se ponían de acuerdo para iniciar el proceso, se buscaba personalmente o se contrataba a un zahorí para marcar el punto más adecuado para realizar la excavación, si el lugar determinado pertenecía a sus fincas, se segregaba de las mismas y se hacía una escritura de compra de la parcela a nombre de los promotores, y si el punto determinado estaba fuera de sus propiedades procedían a la compra mancomunada del terreno a su propietario. Una vez convencidos del posible éxito de la excavación, acudían al notario para constituir la sociedad. 
Las Sociedades Civiles de Regantes, se rigen por unos Estatutos que recogen el objeto de la misma, el capital social, los derechos y obligaciones de los socios, el gobierno y la administración de la sociedad, los cargos y atribuciones de los mismos, etc. Son estatutos tipo que se repite en todas ellas.

Los órganos de administración son la Junta General que representa a la Sociedad y está formada por todos los socios y la Junta Directiva que está formada por los socios elegidos por la Junta General, que ostenta la representación jurídica de la Sociedad y que está formada por un Presidente, un Secretario, un Tesorero y varios Vocales, esta junta será la encargada de llevar a cabo todas las tareas necesarias para la buena marcha de la sociedad. Especial importancia tiene el Presidente, que además de representar a la sociedad debe encargarse de hacer cumplir los Estatutos y de que se ejecuten los acuerdos tomados por las Juntas General y Directiva.

Una vez realizado con éxito el alumbramiento de aguas y conocido su caudal, se procedía a la captación de nuevos socios en número proporcional a la capacidad de riego del alumbramiento. Lo normal era hacer unas doscientas cincuenta acciones, cada una de las cuales daba derecho a una hora de riego, de esta manera se aseguraban una frecuencia de riego de unos veinte días en los periodos menos favorables.

A continuación había que proceder a:

- Instalar el equipo de bombeo.

- Construir la casa.

- Construir la balsa o depósito de regulación

- $\quad$ Establecer e instalar la red de riego.

La instalación de los equipos de bombeo ha ido evolucionando a lo largo del siglo a medida que estos se iban perfeccionando, empezaron con las bombas accionadas por generadores de vapor o gas pobre, como necesitaban un hogar con chimenea, dieron una imagen típica al edificio, continuaron con las centrífugas accionadas por motores eléctricos y terminaron con las bombas sumergidas con el motor eléctrico adosado formando un único cuerpo y capaces de elevar grandes volúmenes de agua desde grandes profundidades.

La casa durante muchos años tuvo una doble función, por un lado contener pozo y equipo de bombeo que en los primeros tiempos era muy aparatoso y por otro servir de vivienda al motorista y su familia. La figura del motorista fue muy importante cuando los equipos no eran de funcionamiento automático.

La construcción de una balsa de regulación de pequeñas dimensiones, alrededor de un centenar de metros cúbicos, fue una práctica común en los primeros pozos, lo observamos en el Pozo del Sordo, en la masía de Baviera, en los Santos Patronos, en el Tonellet, etc.

Establecer e instalar la red de riego, que podía ser muy sencilla si todas las propiedades estaban agrupadas, y se iba haciendo más complicada a medida que aumentaba la dispersión. En condiciones normales la red de tuberías y válvulas de riego se hacían a cargo de la sociedad que posteriormente las repercutía a los socios proporcionalmente al número de acciones.

En algunos casos, las sociedades que tenían grandes sobrantes de agua, por no haber podido reunir suficientes socios en las proximidades del alumbramiento, se planteaban llegar a zonas más alejadas, en las que no había agua, y ofrecer a estos agricultores la posibilidad de hacerse socios. Es el caso de la Sociedad de San Ramón que condujo el agua desde el barranco a la Foya o Viernes Santo que instaló una tubería desde la Venteta hasta la Foya. 
La forma de operar de las sociedades de riego, era la misma que había venido ejerciéndose durante cientos de años en el riego tradicional, consistía en establecer el turno de riego desde la primera hasta la última de las propiedades, el socio que quería regar cuando pasara el agua por su finca, lo pedía con tiempo, y el que no aprovechaba la ocasión debía esperar hasta el próximo turno o tanda.

El precio del agua lo establecía cada año la Junta General a propuesta de la Junta Directiva que una vez contemplado el estado de cuentas de la sociedad y atendiendo a los compromisos adquiridos por la misma, establecía un aumento de precio o una derrama entre los socios. El precio se establecía por el tiempo en horas o fracciones, entendiendo que era más sencillo el control y que el caudal era regular.

Cada sociedad tenía dos empleados fijos que eran el motorista y el regador, el primero se encargaba de la estación de bombeo, puesta en marcha, paro, mantenimiento, limpieza, balsa, etc, tenía la obligación de vivir en la casa y atender a la vigilancia y protección del conjunto. El regador era el encargado de encauzar el agua por tuberías y acequias, abriendo y cerrando válvulas y compuertas y contar el tiempo que cada socio la utilizaba en su propiedad, pasando el parte correspondiente para la facturación. En ocasiones los socios les pedían que se encargaran del riego de la finca a cambio de una gratificación.

Cuando en los años ochenta se inició el nuevo sistema de riego localizado, que fue sustituyendo al antiguo riego por gravedad, las sociedades tuvieron que plantearse la necesidad de grandes inversiones. El nuevo sistema de riego exigía una estación de bombeo que generase una presión mínima de $2 \mathrm{Kg} / \mathrm{cm}^{2}$, en el punto más alejado, un equipo de filtrado y una red de tuberías de presión.

Además, en el caso de optar por un sistema de riego a la demanda, había que asegurar grandes caudales de suministro por lo que había que sobredimensionar el equipo de bombeo y equiparlo de automatismos reguladores de caudal o bien proceder a la construcción de balsas o depósitos elevados de dimensiones notables.

Con las ayudas que proporcionó la Administración, muchas de estas sociedades optaron por el cambio que además de la publicitada ventaja del ahorro de agua, suponía la automatización del riego. Sociedades como San Luís Beltrán, San Francisco, San Paulino, Santos Patronos, etc, procedieron a la construcción de estos depósitos que situaron en puntos elevados de su entorno, de manera que las conducciones de carga y descarga de los mismos no fueran excesivamente largas.

Las sociedades se encargaban de llevar un conducto de presión hasta cada propiedad, y los socios se encargaban de realizar una caseta para su cabezal de riego, en la que se situaba el contador de caudal, filtros, abonadoras etc.

El nuevo sistema hizo variar el antiguo concepto de tarifa horaria por el nuevo de tarifa por metro cúbico consumido.

Las sociedades que se constituyeron a partir de esta época, la Sierra y los Xils, desde su inicio adoptaron este sistema y como el emplazamiento de los alumbramientos tenía cerca zonas elevadas, construyeron en ellas sus depósitos, la Sierra en la ladera sur de Perenchiza y los Xils junto al camino de Barbeta. Como sus caudales eran grandes los depósitos fueron de grandes dimensiones.

Muchas sociedades por sus características particulares no se apuntaron al cambio de sistema de riego y han continuado practicando el tradicional. Son en su mayoría las que riegan la huerta antigua como San Cristóbal, San Jaime, San Roque, San Ramón, Santa Rita, San Jorge, etc. El tamaño de las propiedades de los socios son pequeñas, sus cultivos suelen ser de hortalizas y sus hábitos de cultivo no tienen ningún deseo de cambiarlos. Además 
son zonas muy presionadas por el urbanismo que desincentivan la producción agrícola. Para agravar más la situación de estas sociedades se vienen produciendo en los últimos años hurtos y vandalismos en sus solitarias instalaciones que les producen pérdidas irreparables que ponen en entredicho su continuidad.

\section{Evolución de la superficie de regadío}

Partiendo de la información gráfica digitalizada hemos realizado el estudio de los cambios observados en el patrón de parcelación, una vez calculadas las áreas de cada una de las parcelas catastrales. Con cálculos simples estadísticos univariantes como la media y la desviación típica aplicados a las superficies de las parcelas es posible comprobar si los cambios en la situación agronómica incluyen reagrupación parcelaria y, tanto la tabla como las figuras así lo indican claramente.

Tabla 3. Estudio de parcelación.

\begin{tabular}{|c|c|c|c|c|c|}
\hline \multicolumn{6}{|c|}{ Número de parcelas agrícolas } \\
\hline \multicolumn{2}{|c|}{1900} & \multicolumn{2}{|c|}{1955} & \multicolumn{2}{|c|}{2009} \\
\hline regadío & secano & regadío & secano & regadío & secano \\
\hline 1.583 & 7.795 & 3.513 & 5.798 & 4.693 & 1.362 \\
\hline \multicolumn{6}{|c|}{ Area total de la superficie dedicada al cultivo ( $\mathrm{m} 2)$} \\
\hline \multicolumn{2}{|c|}{1900} & \multicolumn{2}{|c|}{1955} & \multicolumn{2}{|c|}{2009} \\
\hline regadío & secano & regadío & secano & regadío & secano \\
\hline 3.753 .900 & 52.231 .539 & 14.461 .462 & 41.467 .937 & 33.828 .243 & 9.560 .444 \\
\hline \multicolumn{6}{|c|}{ Area media de las parcelas dedicadas al cultivo $(\mathrm{m} 2)$} \\
\hline \multicolumn{2}{|c|}{1900} & \multicolumn{2}{|c|}{1955} & \multicolumn{2}{|c|}{2009} \\
\hline regadío & secano & regadío & secano & regadío & secano \\
\hline 2.371 & 6.701 & 4.117 & 7.152 & 7.208 & 7.019 \\
\hline \multicolumn{6}{|c|}{ Desviación típica de la superficie de las parcelas dedicada al cultivo (m2) } \\
\hline \multicolumn{2}{|c|}{1900} & \multicolumn{2}{|c|}{1955} & \multicolumn{2}{|c|}{2009} \\
\hline regadío & secano & regadío & secano & regadío & secano \\
\hline 3.166 & 9.671 & 6.238 & 10.256 & 14.663 & 31.203 \\
\hline
\end{tabular}

Tabla 4. Evolución superficie de regadío y de secano.

\begin{tabular}{|c|c|c|c|c|c|c|}
\hline \multirow[t]{2}{*}{ Cultivos } & \multicolumn{2}{|l|}{1900} & \multicolumn{2}{|l|}{1955} & \multicolumn{2}{|l|}{2000} \\
\hline & $\begin{array}{l}\text { Superficie en } \\
\text { hanegadas }\end{array}$ & $\%$ & $\begin{array}{c}\text { Superficie en } \\
\text { hanegadas }\end{array}$ & $\%$ & $\begin{array}{c}\text { Superficie en } \\
\text { hanegadas }\end{array}$ & $\%$ \\
\hline Secano & 65.096 & 94,1 & 51.892 & 75,4 & 9.751 & 18,4 \\
\hline Regadío & 4.031 & 5,9 & 16.899 & 24,6 & 43.084 & 81,6 \\
\hline TOTAL & 69.127 & & 68.790 & & 52.835 & \\
\hline
\end{tabular}




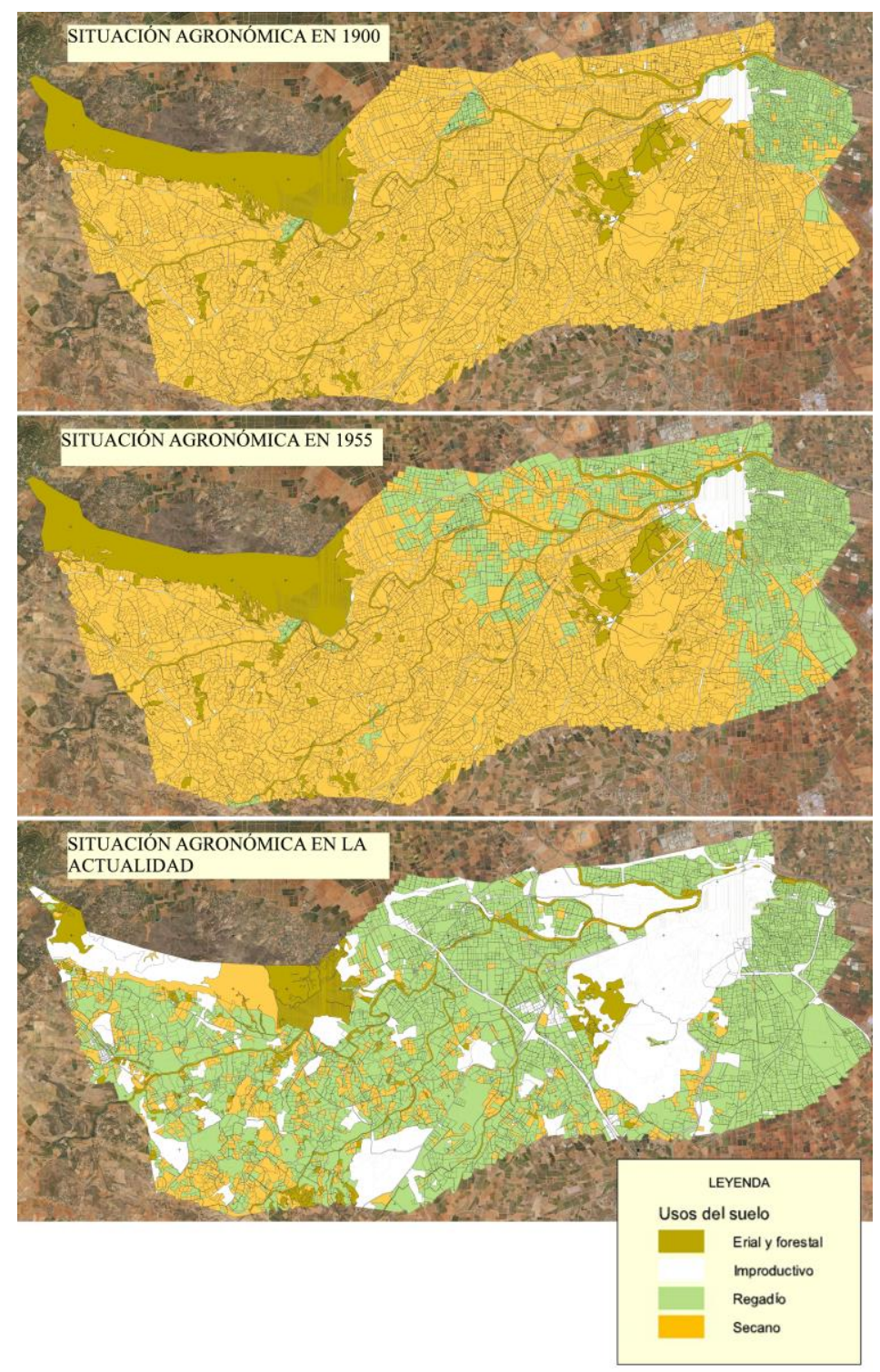

Fig. 4. Evolución de la superficie cultivada.

\section{Referencias}

Beguer Esteve, V. (1977): Obra Completa, Volumen II. Edita, Ajuntament de Torrent.

Burriel de Orueta, E. (1970) La buerta de Valencia zona sur. Edita Institución Alfonso El Magnánimo. 1.970.

Cavanilles, AJ. (1795): Observaciones sobre la Historia natural, geografía, agricultura, población y frutos del Reyno de V alencia, Madrid, Imprenta Real.

Congreso Nacional de Riegos (1921): III Congreso Nacional de Riegos, Editorial Hijos de F. Vives Mora. Valencia.

Folgado García, P. (1994): Propietat agraria i paisatge rural durant la segona meitat del segle XIX a Torrent. Torrens, Estudis $i$ Investigacions de Torrent $i$ Comarca. Publicació de l'arxiu, biblioteca i museu de l'Ajuntament de Torrent.

Fresquet Gozalvo, J. (2012): Estudi de l'evolució del sector Agrari a Torrent al llarg del segle XX. Editorial Universitat Politècnica de València. 
Madoz, P. (1845): Diccionario geográfico - estadístico - bistórico de Alicante, Castellón y Valencia. Edita Instituçió “Alfons el Magnànim”.

Maroto Borrego, J. V. (1988): Historia de la Agronomía. Ediciones Mundi-Prensa.

Royo Martinez, J. (2006): Torrent en la prensa de Valencia. La voz de Valencia (1901 - 1917). Edita Ayuntamiento de Torrent.

Royo Martinez, J. (2009): Torrent en la prensa de Valencia. Diario de Valencia (1911 - 1936). Edita Ayuntamiento de Torrent.

Vicent, S.; Almerich, J.M. (2003): Els Pous de Torrent. Ayuntamiento de Torrent, Valencia. 